Mathematical and Computational Applications, Vol. 16, No. 3, pp. 630-640, 2011.

(C) Association for Scientific Research

\title{
MODELLING THE INTERACTION BETWEEN WATER WAVES AND THE OSCILLATING WATER COLUMN WAVE ENERGY DEVICE
}

\author{
Utku Şentürk, Aydoğan Özdamar \\ Mechanical Engineering Department, Faculty of Engineering, Ege University, 35100 \\ Bornova, Izmir, Turkey, aydogan.ozdamar@ege.edu.tr
}

\begin{abstract}
The interaction between regular water waves and the oscillating water column (OWC) geometry is presented in this work. The OWC has a vertical cylindrical geometry, with walls of negligible thickness. Water waves are generated in a numerical wave tank, with the commercial CFD code Fluent, where the dynamic mesh feature and the VOF Model is used for wave generation and the water-air interaction, respectively. The flow is assumed to be viscous, unsteady and incompressible for the numerical model. To focus on the flow inside, 3D, single phase modelling of the OWC device itself is handled by adopting a piston-like pumping flow, using the free surface elevation data found in the former model as an input. To validate the results, a simplified theoretical model of a wave energy converter, namely the Japanese multiOWC barge Kaimei is used. The theoretical model is based on the theory of a compressible air flow where the free surface elevation is assumed to be one dimensional.
\end{abstract}

Key Words- Ocean Wave Energy, Oscillating Water Column, Computational Fluid Dynamics, Numerical Wave Tank.

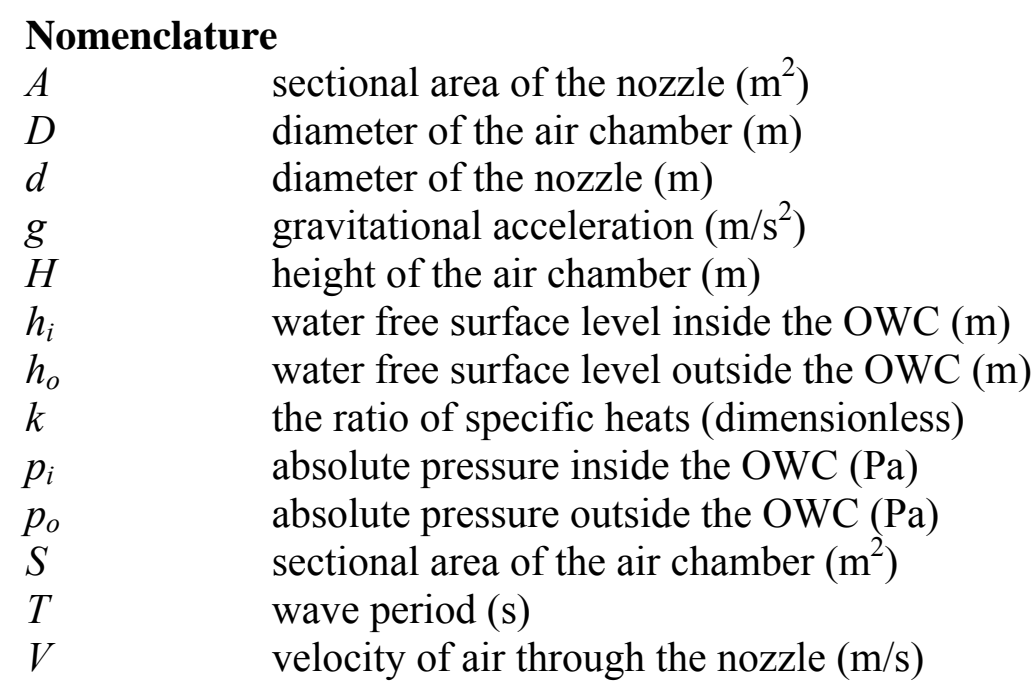

\section{Greek Letters}

$\gamma_{i}$

$\gamma_{o}$

$\gamma_{w}$

$\zeta$

$\Lambda_{i}$

$\Lambda_{o}$

$\omega$ specific weight of air inside the OWC $\left(\mathrm{N} / \mathrm{m}^{3}\right)$ specific weight of air outside the OWC $\left(\mathrm{N} / \mathrm{m}^{3}\right)$ specific weight of water $\left(\mathrm{N} / \mathrm{m}^{3}\right)$

wave amplitude (m)

the exhaust air volume released from inside $\left(\mathrm{m}^{3}\right)$

the exhaust air volume released to outside $\left(\mathrm{m}^{3}\right)$

circular frequency $\left(\mathrm{s}^{-1}\right)$ 


\section{INTRODUCTION}

The global power potential represented by waves that hit all coasts worldwide, has been estimated to be in the order of $1 \mathrm{TW}\left(1\right.$ terawatt $\left.=10^{12} \mathrm{~W}\right)$. If wave energy is harvested on open oceans, energy that is otherwise lost in friction and wave breaking, may be utilised. Then the global wave-power input is estimated to be one order of magnitude larger $\left(\sim 10^{13} \mathrm{~W}\right)$, a quantity that is comparable with the world's present power consumption [1].

Amongst the numerous devices that have been suggested for wave energy conversion, the oscillating water column (OWC) wave energy device is probably the most extensively studied type. Several studies have been carried out by researchers on the theory of the flow inside the oscillating water column (OWC) as well as the energy conversion stage. A theoretical analysis of the power generated by a pneumatic type wave energy conversion buoy was presented by M. E. McCormick where the heaving motion is included since the energy conversion buoy floats [2]. A. Hiramoto [3] studied the theoretical analysis of an air turbine generation system that is not affected by the water waves and remains stationary which particularly forms the underlying principles for the theoretical model of this paper. In another early publication, Evans [4] investigated the OWC performance, treating it as a boundary value problem with the extensive use of matched asymptotic expansions and the linear wave theory. Sarmento and Falcão [5] studied the OWC problem including the linear and nonlinear turbine characteristics. Evans [6] presented the general results for systems of OWCs using the concepts of wave diffraction and radiation and introduced some new reciprocal relations . Later, Porter and Evans presented the efficient calculation of hydrodynamic properties and performance of 2D [7] and cylindrical 3D [8] OWC devices. T.H. Kim et al. [9] and T. Setoguchi et al. [10] focused on the energy conversion stage of an OWC regarding Wells turbines and modifications related to the airflow velocities. A. F. De O. Falcão [11] applied a stochastic model to devise an optimal algorithm for the rotational speed control of an OWC wave power plant equipped with a Wells turbine and to evaluate the average power output. A. El Marjani et al. [12] and Paixãdo Conde and Gato [13] presented work devoted to the numerical modelling in wave energy conversion systems where the flow characteristics of the OWC system were predicted based on the same manner of the 3D flow simulations of this paper.

Modelling the interaction between water waves and an OWC, that is, geometrically 2 concentric cylinders -the air chamber \& the nozzle section- subjected to a train of regular waves is the scope of this study. The theoretical analysis that was given by A. Hiramoto [3] which is one of the early and rather simplified theories forms the underlying principles of the validation section of this paper. Thus the main purpose of the paper is to investigate CFD capabilities for the OWC problem which will cover the groundwork for more complicated theories such as those, pioneered by Evans.

The layout of the paper is as follows. Section 2 introduces the OWC model that is under consideration and presents the theoretical model. The method of the numerical simulations is also given in detail. In Section 3, overall results were presented and discussed such as the analytical results that are found using the theory that is given in subsection 2.2 and numerical results obtained using the method given in subsection 2.3. Section 4 presents the concluding remarks of this study. 


\section{MODELLING}

The oscillating water column device problem that is under consideration is introduced and the models that are adopted to tackle the problem are given in the following subsections.

\subsection{The oscillating water column}

The OWC device bounds an air chamber where the air interacts with the water free surface that is disturbed by outer water waves. The air flow caused by the inner water level oscillation has two cases: Inhalation and exhalation (Fig. 1).

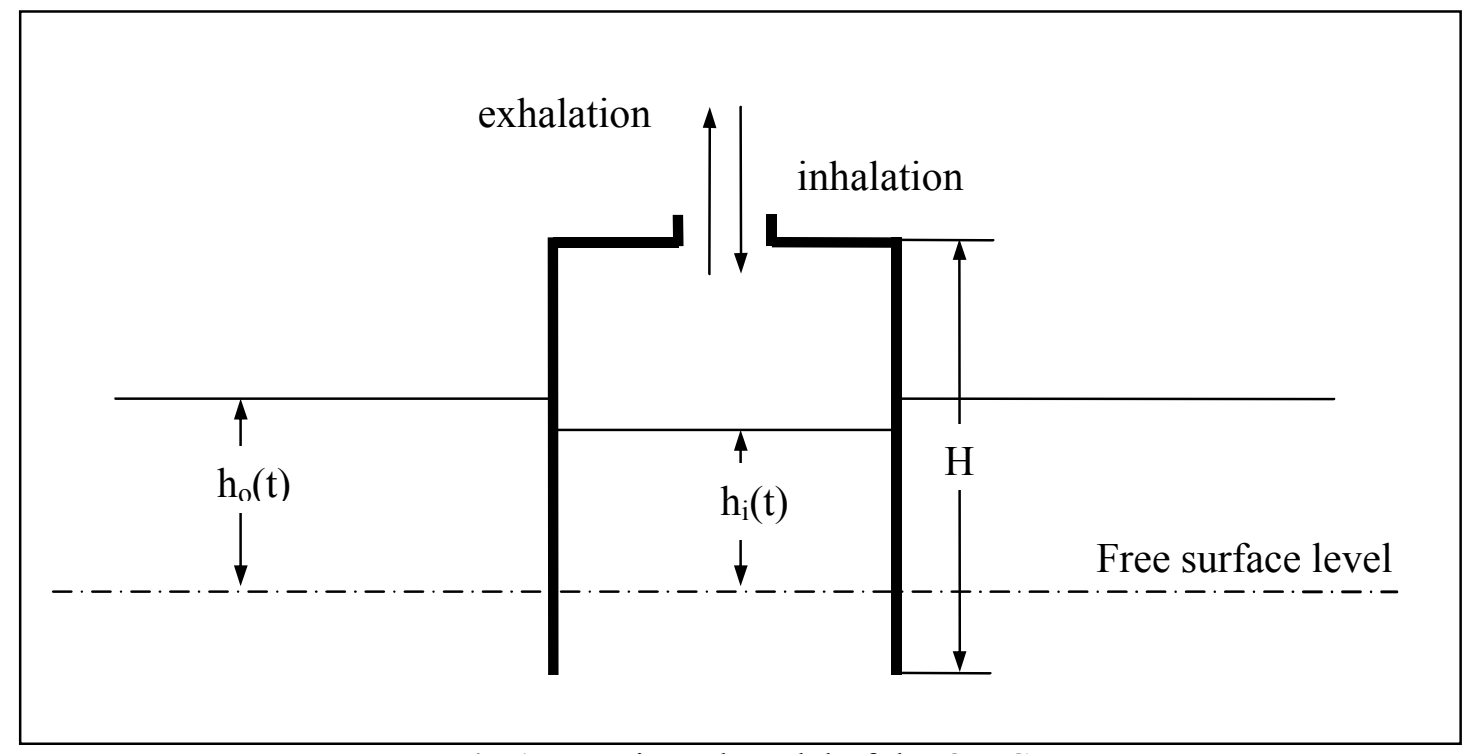

Fig.1 Investigated model of the OWC

Investigated case in this paper is an OWC partially submerged in water which is exposed to regular waves with an amplitude of $\zeta=1.2 \mathrm{~m}$ and period of $T=5 \mathrm{~s}$. Linear wave theory is used for the wave elevation function $h_{o}(t)$ of the outside waves. The OWC is assumed to be moored restricting the heaving motion. The height $(H)$ and the diameter $(D)$ of the air chamber are both $10 \mathrm{~m}$ and the diameter of the nozzle $(d)$ is $1 \mathrm{~m}$. The absolute pressure outside is assumed to be $p_{0}=10^{5} \mathrm{~Pa}$. The specific weights of water and air are $\gamma_{w}=10^{4} \mathrm{~N} / \mathrm{m}^{3}$ and $\gamma_{o}=12 \mathrm{~N} / \mathrm{m}^{3}$. The ratio of the specific heats is $k=1.4$.

\subsection{Theoretical model}

The theoretical model that was given by Hiramoto is adopted exactly. Using Bernoulli's theorem, following relation holds for the inner water level and the air pressure provided that the air chamber remains stationary.

$h_{i}+\frac{p_{i}}{\gamma_{w}}=h_{o}+\frac{p_{o}}{\gamma_{w}}$

Assuming the air inside the chamber is compressible and isentropic perfect gas [14],

$\frac{p_{i}}{\gamma_{i}^{k}}=\frac{p_{o}}{\gamma_{o}^{k}}$ 
can be written. Again, using Bernoulli's theorem, the velocity of the exhaust air through the nozzle $V$, can be written as follows:

$\frac{V^{2}}{2 g}+\left(\frac{k}{k-1}\right)\left(\frac{p_{o}}{\gamma_{o}}\right)=\left(\frac{k}{k-1}\right)\left(\frac{p_{i}}{\gamma_{i}}\right)$

In the case of suction, it is possible to write,

$\frac{V^{2}}{2 g}+\left(\frac{k}{k-1}\right)\left(\frac{p_{i}}{\gamma_{i}}\right)=\left(\frac{k}{k-1}\right)\left(\frac{p_{o}}{\gamma_{o}}\right)$

Using Eq. 1,2 for $p_{i} / \gamma_{i}$ and substituting into Eq. 3a gives,

$V^{2}=2 g\left(\frac{k}{k-1}\right)\left(\frac{p_{o}}{\gamma_{0}}\right)\left[\left(1+\frac{\left(h_{0}-h_{i}\right) \gamma_{W}}{p_{0}}\right)^{\left(\frac{k-1}{k}\right)}-1\right]$

Now, substituting into Eq. 3 bives,

$V^{2}=2 g\left(\frac{k}{k-1}\right)\left(\frac{p_{o}}{\gamma_{0}}\right)\left[1-\left(1+\frac{\left(h_{0}-h_{i}\right) \gamma_{w}}{p_{0}}\right)^{\left(\frac{k-1}{k}\right)}\right]$

After approximations and substitutions, for $g, p_{o}, \gamma_{0}, \gamma_{w}$ and $k$, Eq. $4 \mathrm{a}$ and $4 \mathrm{~b}$ are combined into a more convenient form [3],

$V=127.8 \sqrt{\left|h_{0}-h_{i}\right|}$

It is now possible to calculate the air flow velocity $V$ from Eq. 5, provided that the inner water level $h_{i}$ is known.

Some part of the air in the chamber flows out through the nozzle sectional area $A$ at the pressure $p_{0}$ and the velocity $V$, while the pressure and the volume of the chamber turns from $p_{i}(t)$ and $S \times\left[H-h_{i}(t)\right]$ to $p_{i}(t+\Delta t)$ and $S \times\left[H-h_{i}(t+\Delta t)\right]$ where $\mathrm{S}$ is the sectional area of the air chamber. Then, the exhaust air volume released to outside is,

$$
\Lambda_{o}=A \times V \times \Delta t \text {, at } p_{0}
$$

On the other hand, using the continuity equation for compressible flow through the nozzle and Eq. 2, the exhaust air volume released from inside is,

$$
\Lambda_{i}=\left[\frac{p_{i}}{p_{i}(t+\Delta t)}\right]^{1 / k}(A \times V \times \Delta t), \quad \text { at } p_{i}(t+\Delta t)
$$

Assuming that the air mass is conserved for the given air chamber between times $t$ and $t+\Delta t$, and using Eq. 2, following relation can be obtained.

$$
p_{1}(t)\left\{S \times\left[H-h_{i}(t)\right]\right]^{k}=p_{1}(t+\Delta t)\left\{S \times\left[H-h_{i}(t+\Delta t)\right]+\left[\frac{p_{0}}{p_{i}(t+\Delta t)}\right]^{1 / k}(A \times V \times \Delta t)\right\}^{k}
$$

By using Eq. 2, 4a, 4b and Eq. 8 as $\Delta t \rightarrow 0$, the final form of the equations, after approximation and substitution of known values are [3],

$\left(H+14+1.4 h_{0}-2.4 h_{i}\right) \frac{d h_{i}}{d t}-\left(H-h_{i}\right) \frac{d h_{0}}{d t}=1790 \frac{A}{\xi}\left[1+0.0286\left(h_{0}-h_{i}\right)\right] \sqrt{h_{0}-h_{i}}$

, when $h_{0} \geq h_{i}$

$\left(H+14+1.4 h_{0}-2.4 h_{i}\right) \frac{d h_{i}}{d t}-\left(H-h_{i}\right) \frac{d h_{0}}{d t}=-1790 \frac{A}{5}\left[1+0.1\left(h_{0}-h_{i}\right)\right] \sqrt{h_{i}-h_{8}}$, when

$h_{i} \geq h_{0}$

Assuming that the outer water level function $h_{o}(t)$ is known, the inner water level function $h_{i}(t)$ can be given as a solution of these equations where Eq. 9a and Eq. 9b are distinct definitions of exhalation and inhalation cases, respectively. The square root 
terms clearly state that, $\mathrm{Eq} 9 \mathrm{a}$, and $\mathrm{Eq} 9 \mathrm{~b}$ do not have a real solution outside the defined intervals.

Using Linear Wave Theory [15] for regular waves, outer water level $h_{o}(t)$ is assumed to be

$$
h_{0}(t)=\zeta \sin \omega t
$$

where $\zeta$ is the wave amplitude and $\omega=2 \pi / T$ is the circular frequency.

In this study, a computer code is developed in Matlab 7.0. Solutions sets for Eq. 9a and Eq. $9 \mathrm{~b}$ are derived using Matlab's ode 23 solver, spanning a time of 1 period $(T=5$ s). The code switches between these two, until the active equation returns a complex number, extracting the $h_{i}$ value and the jump step that define the initial conditions for the other differential equation to be solved. Evaluating the collected $h_{o}$ and $h_{i}$ values into Eq. 5, returns the air flow velocity through the nozzle.

\subsection{Numerical Simulations}

The numerical modelling involves CFD analyses of the OWC problem. The commercial code Fluent 6.2 is used which solves the Navier-Stokes equations numerically based on Finite Volume Method approach. The flow is assumed to be viscous, unsteady and incompressible for the numerical model. Water waves are generated in a numerical wave tank, where Fluent's dynamic mesh feature and the VOF Model are used for wave generation and the water-air interaction, respectively.

The numerical analyses involve two steps. First, a wave tank is modeled in 2D. Water waves are generated using Fluent's dynamic mesh feature and VOF Model is used for water-air interaction [16]. The water is initially at rest and wall type boundary condition is assigned to the OWC walls as well as the problem domain boundaries except the upper wall is defined as pressure outlet boundary with zero gage pressure (Fig. 2). The inner water level $h_{i}(t)$ is found to be in good agreement with the results acquired using the theoretical model given in above, while the air flow velocity variation through the nozzle is incompatible. For this reason, to focus on the flow inside the OWC, the second step includes the numerical modelling of the OWC device itself, single phase, in 3D by adopting a piston-like pumping flow using the $h_{i}(t)$ found in the second step as an input. This step returns improved results for the air flow velocity through the nozzle. 


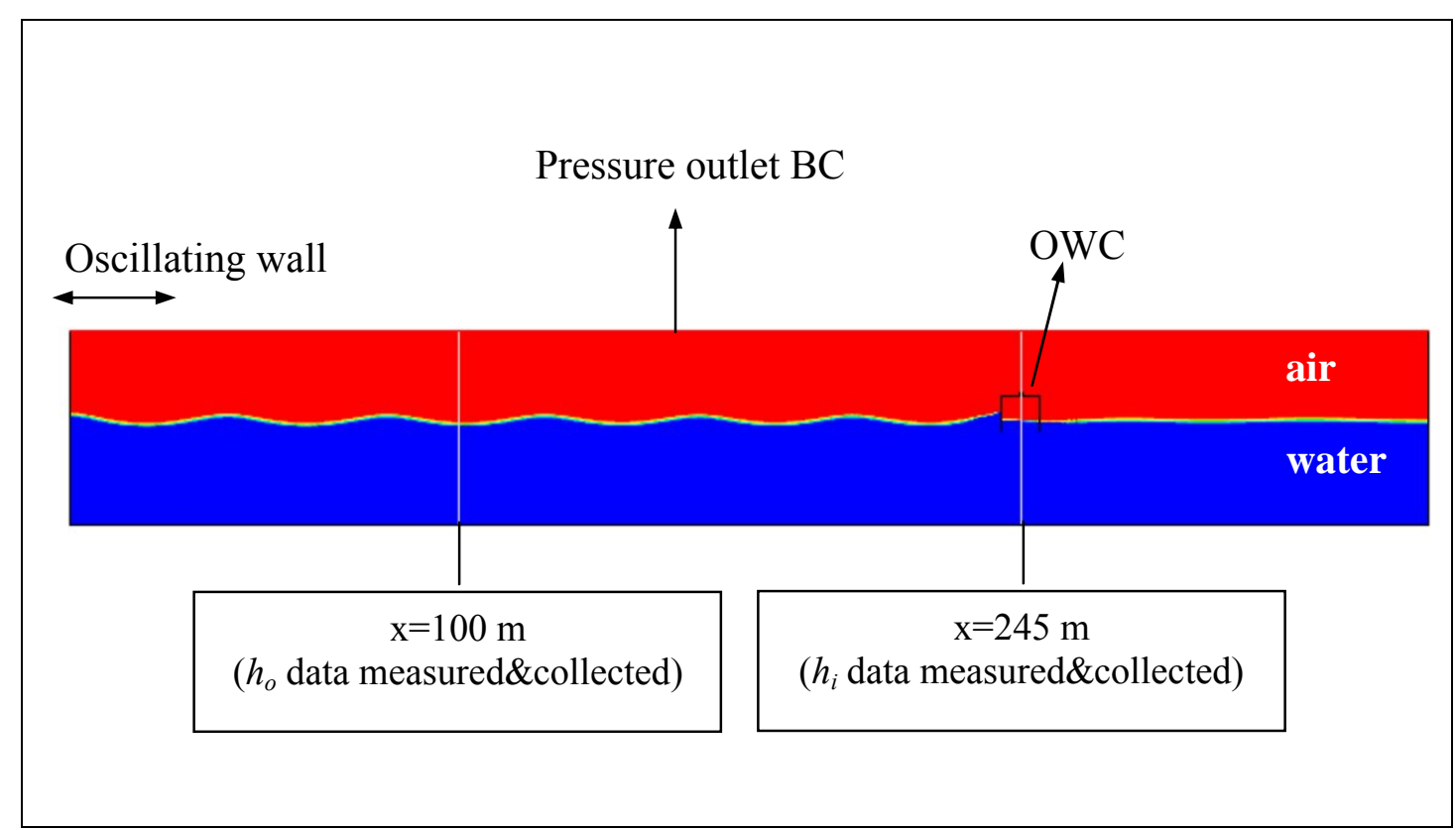

Fig.2 CFD model of the wave tank and the OWC in 2D

The wave tank and the OWC are modelled by using Fluent's preprocessor Gambit according to the given dimensions in subsection 2.1 (Fig. 2). The model is then uniformly meshed with quad elements with a total mesh size of 70000 (Fig. 3a). The sinusoidal motion of the left, wall type boundary of the wave tank is assigned using a user defined function (UDF) [16]. The non-iterative time advancement (NITA) scheme is used. For all cases, time step size is fixed to 0.005 s. 25000 time steps were completed ending up with $125 \mathrm{~s}$ of data output.

To investigate the grid independency, a finer, hybrid mesh is generated around the OWC with a total mesh size of 89726 (Fig. 3b). The inner water level $h_{i}(t)$ results are given in Figure 7 of both cases. Since good accuracy is achieved in the results, no further grid refinement is handled.

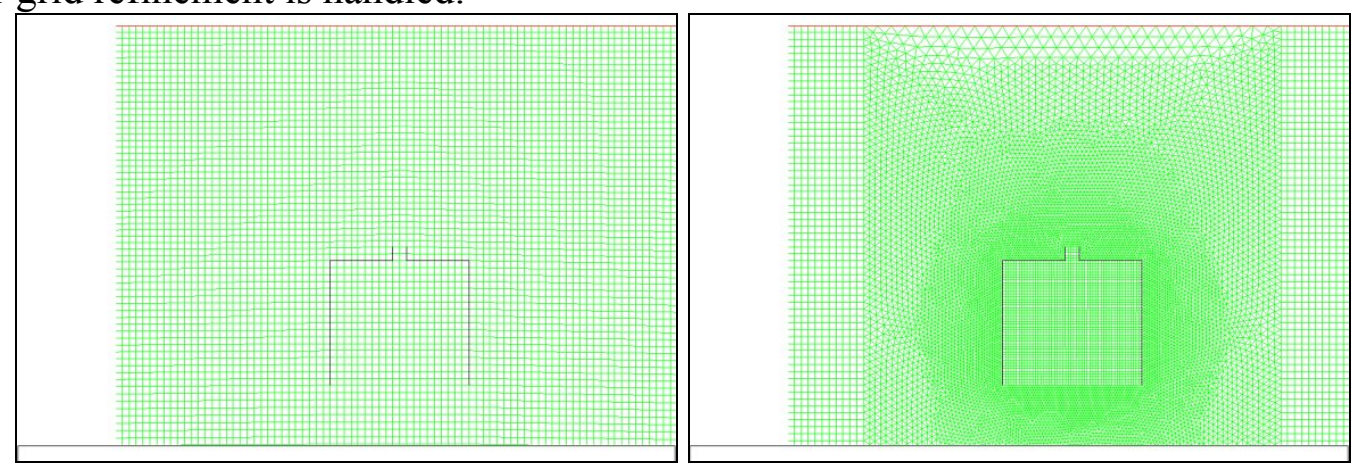

Fig.3 (a) Uniform quad mesh around the OWC (b) Hybrid mesh around the OWC

To analyze the air flow inside the air chamber and the nozzle section, the OWC is modelled in 3D. This time, the bottom wall is assumed to be moving and a hybrid mesh is generated with a total size of 79251 and maximum skewness of 0.82 (Fig 4). The nozzle section end of the model is assigned as an outlet boundary where the area- 
weighted average of velocity values are captured for each time step since they are the required for the post-processing stage. Iterative time advancement scheme is used where all the equations are solved iteratively, for a given time-step, until the convergence criteria are met to avoid possibility of divergence eliminating the splitting error [17].

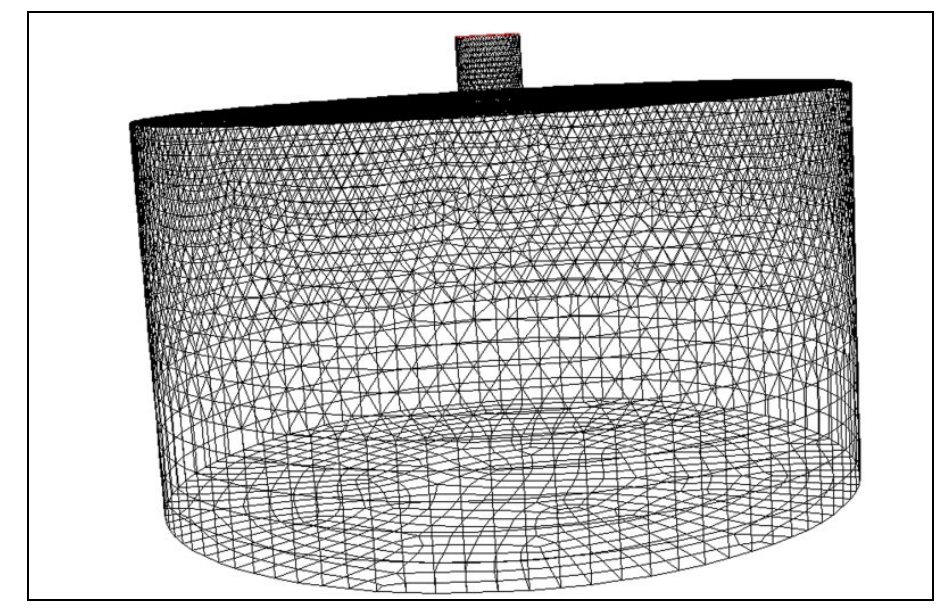

Fig.4 CFD model of the wave tank and the OWC in 2D

\section{RESULTS AND DISCUSSION}

Water levels inside $\left(h_{i}\right)$ and outside $\left(h_{o}\right)$ the OWC acquired using the theoretical model described in subsection 2.2 are given in Fig. 5 for one period of time.

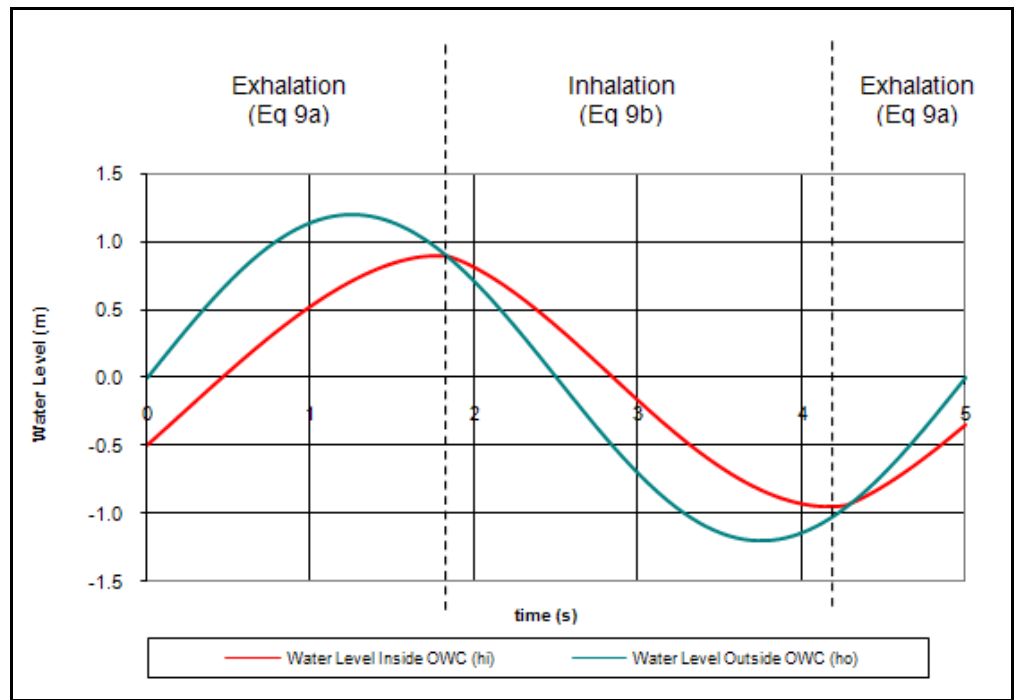

Fig.5 Water levels inside $\left(h_{i}\right)$ and outside $\left(h_{o}\right)$ the OWC acquired using the analytical model

Fig. 6a and Fig. 6b show $h_{i}$ and $h_{o}$ oscillations for the time spanned numerically using CFD. The free surface evolution pattern outside the OWC follow a sinusoidal form with the same angular frequency assigned to the moving wall boundary which acts as a wave generator. Fig. 6a shows a smaller time interval for a more expanded view, while Fig. $6 \mathrm{~b}$ gives the elevation versus total time spanned. 


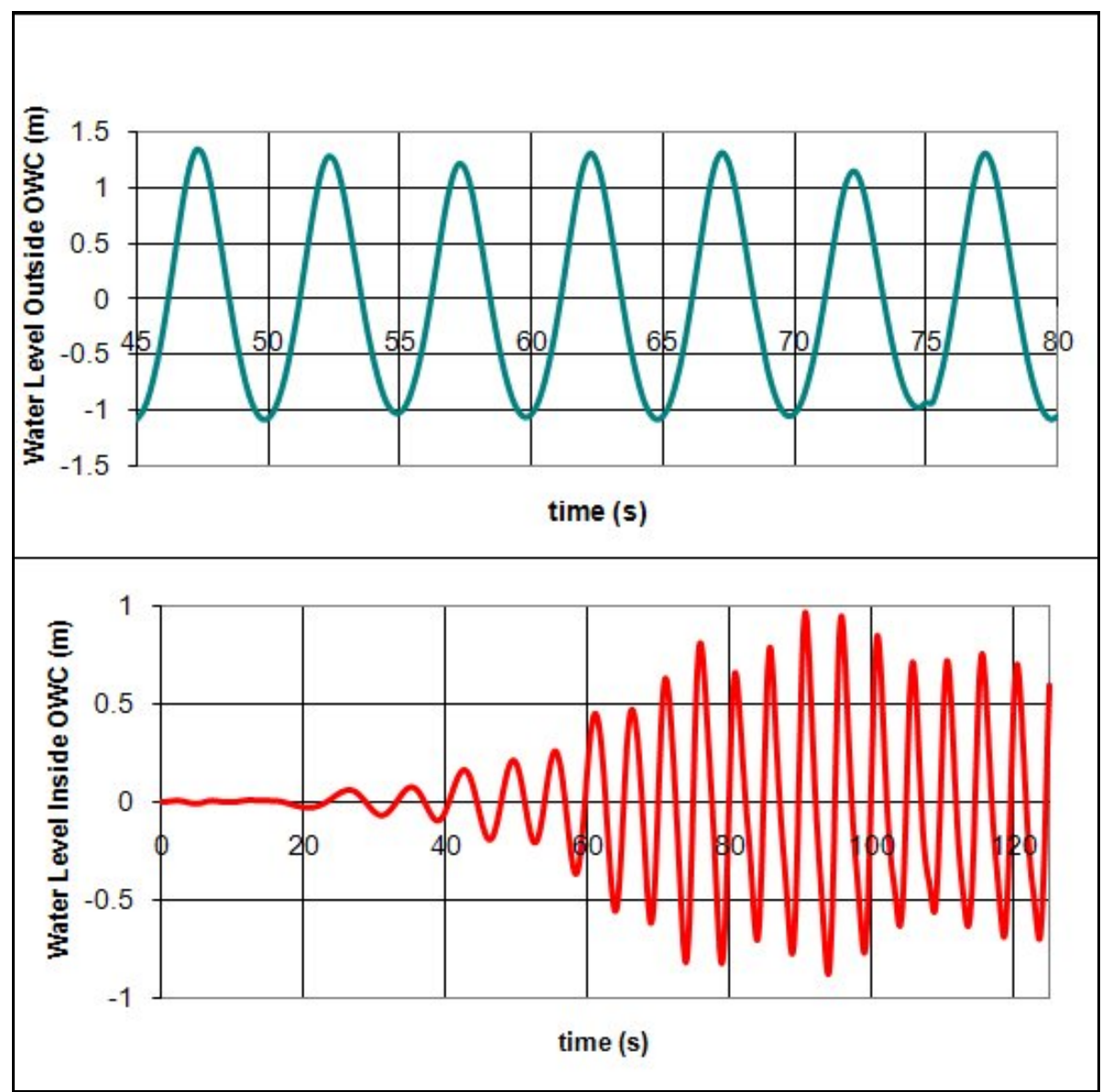

Fig.6 Numerical results: (a) Water level outside the OWC (b) Water level inside the OWC

Grid independency investigation compares two sets of $h_{i}$ values from uniform quad meshed and the finer, hybrid meshed models are given in Figure 7 for a time interval of a single period. Elevation data are found to be in good agreement and no further grid refinement is handled.

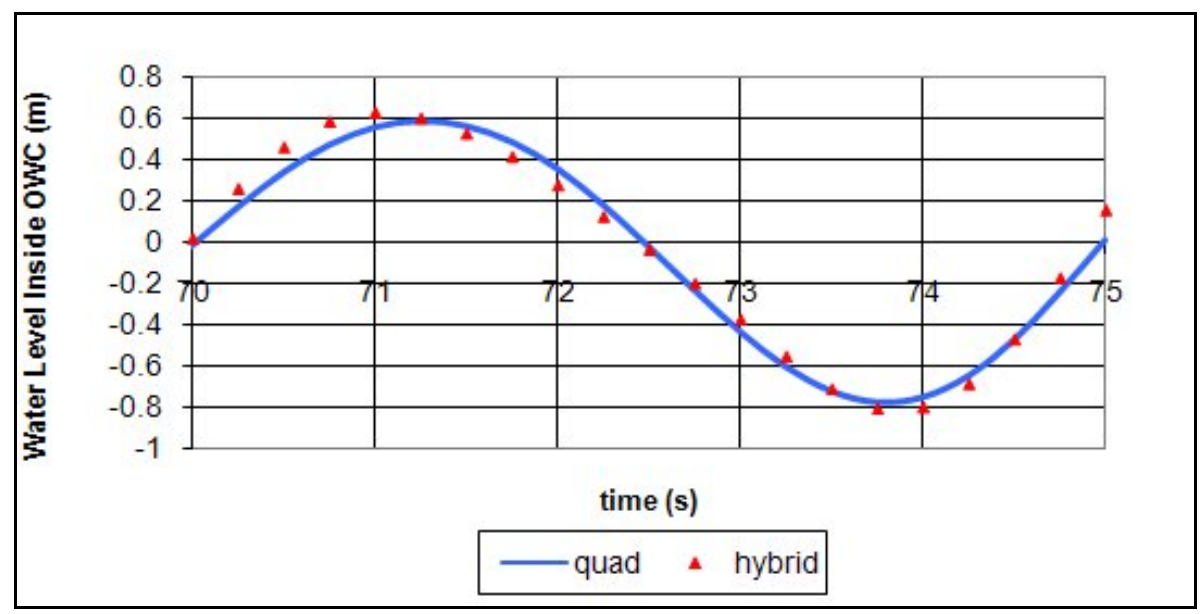

Fig.7 Water level inside OWC-uniform quad and the finer, hybrid meshed models 
Fig. 8a and Fig. 8b show both analytical and numerical results for $h_{i}$ and $h_{o}$ where a slight phase difference is applied to the analytical results by translating the initial condition.

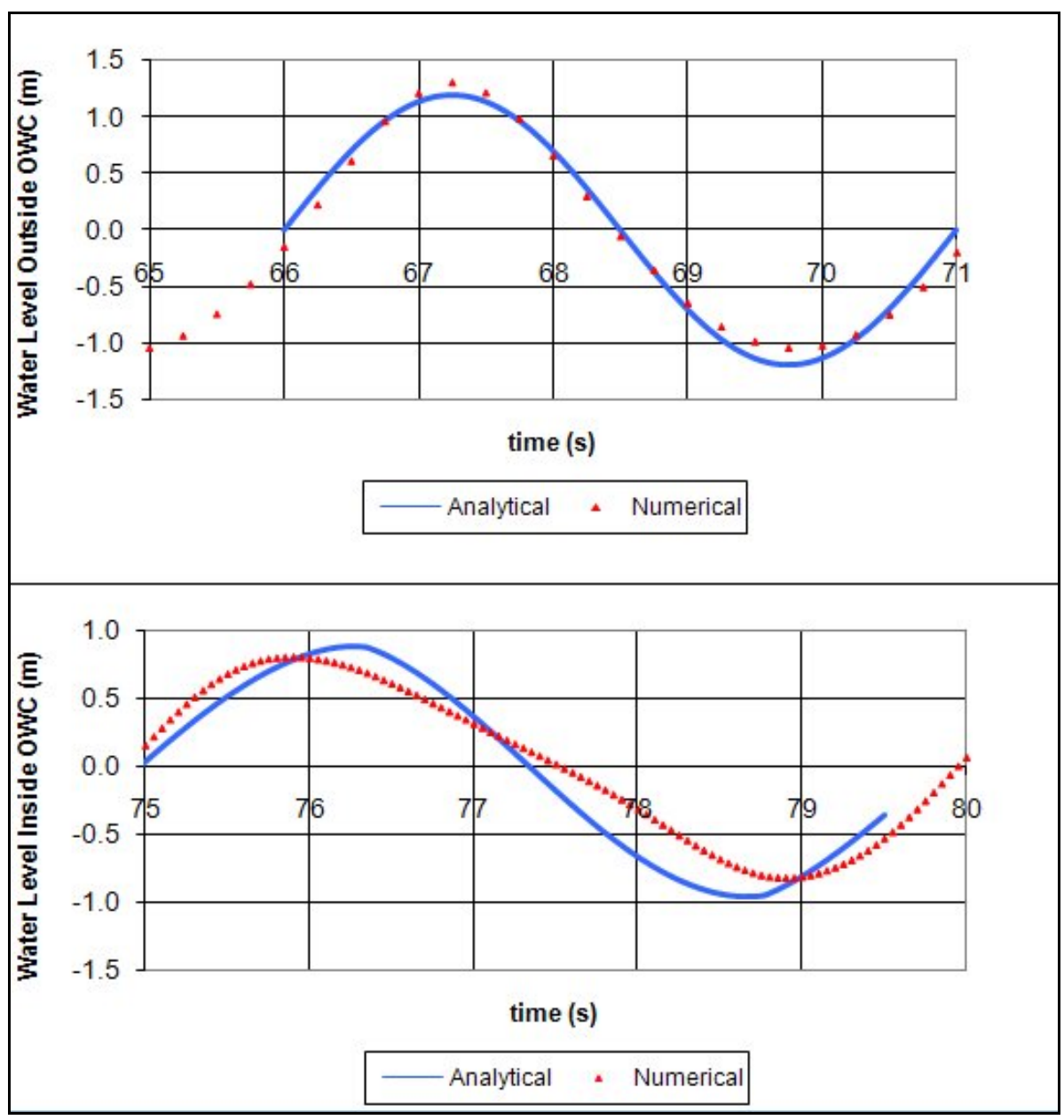

Fig.8 Comparisons: (a) Water level outside the OWC (b) Water level inside the OWC

Fig. 9a shows the velocity at the nozzle section using both analytical and numerical models. 


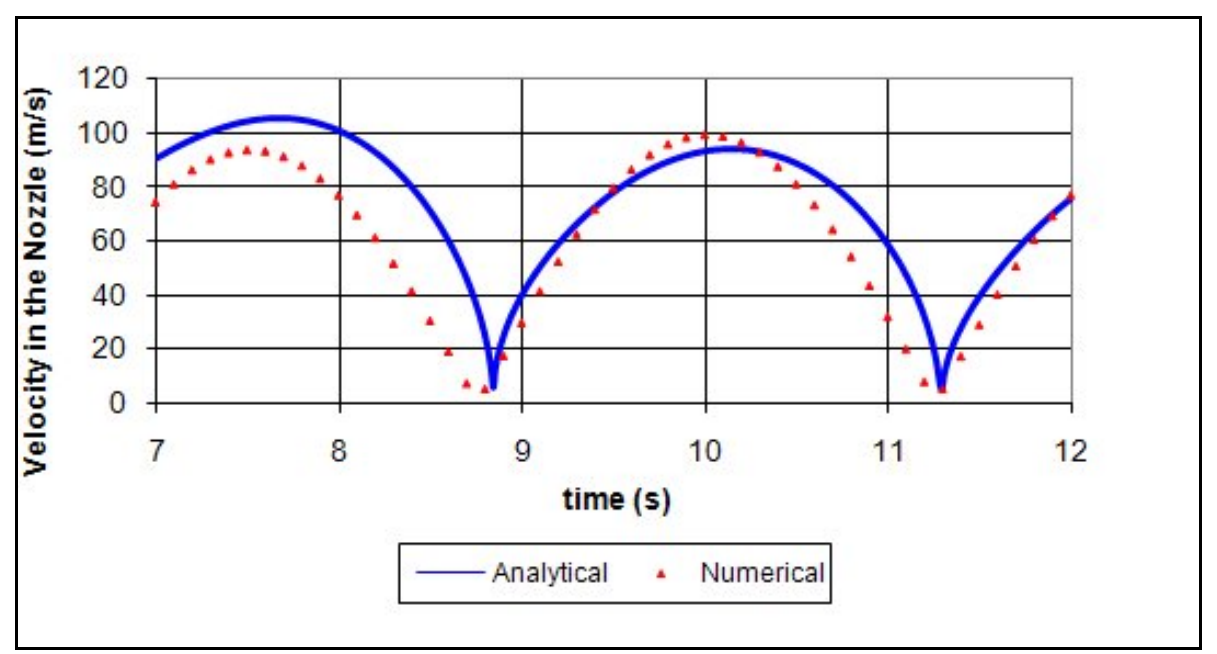

Fig.9 Velocity variation in the nozzle

The differences between the analytical and numerical results arise from the assumptions for the mathematical modelling in both approaches. To summarize, in the analytical approach, it's assumed that the air flow is compressible, the air chamber remains stationary and Bernoulli's theorem is used for the air flow through the nozzle. Thus, the problem is considered one dimensional and the wave profile outside the OWC is assumed to be sinusoidal. In the numerical approach, Volume of Fluid Model feature of the Fluent software is used, so that the flow is governed by incompressible NavierStokes equations where the flow is viscous, and the wave profile is generated by the wall motion of the numerical wave tank. As a result, two dimensional velocity and pressure fields are obtained for both water and air phases. The water level $h_{i}(t)$ is recorded with an imaginary wave probe as given in Fig. 2 which essentially neglects the spatial variations and assumes a piston-like motion.

\section{CONCLUDING REMARKS}

The outer water level $h_{o}(t)$, the inner water level $h_{i}(t)$ and the air flow velocity through the nozzle $V$ of OWC were acquired using the analytical model and the numerical simulations. Concluding remarks can be extracted from this study as follows:

- The analytical results are based on the theory of a compressible air flow neglecting the losses in the fluid-fluid and the fluid-structure interactions while the flow was assumed to be viscous and incompressible for the numerical model. Comparing both models; this resulted in slight differences in $h_{i}(t)$, while up to 30 $\%$ relative error was experienced in the air flow velocity through the nozzle $V$. Investigated case has a sectional area ratio where the air velocity through the nozzle reaches the incompressibility limit, $\mathrm{Ma}=0.3$. Further CFD analyses should include compressibility effects or a proper modification of the area ratio.

- Modelling waves and interactions with structures using FLUENT's VOF Model and dynamic mesh feature was found suitable for further studies.

- More complex theories that were presented in Ref. About OWC hydrodynamics where spatial variations of the free water surface inside the OWC is included should also be investigated in further studies. 
- Possibility of financial support to obtain the equipment for the wave tank experiments reveals the third approach, the experimental method, which will be the next subject of research of this study.

- Obtained results would be used as input for a performance analysis of a Wells turbine [12], that is placed in the nozzle section, approximating the overall efficiency of the OWC device by using CFD.

\section{REFERENCES}

1. Falnes J., A review of wave energy extraction, Marine Structures 20, p. 185-201, 2007.

2. McCormick M. E., Analysis of a wave energy conversion buoy, Journal of Hydronautics 8, p. 77-82, 1974.

3. Hiramoto A., The theoretical analysis of an air turbine generation system, International Symposium on Wave and Tidal Energy, p. 73-84, 1978.

4. Evans D. V., The oscillating water column wave energy device, Journal of the Institute of Mathematics and its Applications, 22, p. 423-433, 1978.

5. Sarmento A. J. N. A. and Falcao A. F. de O. , Wave generation by an oscillating surface pressure and its application in wave energy extraction, Journal of Fluid Mechanics, 150, p. 467-485, 1984.

6. Evans D. V., Wave Power Absorption by Systems of Oscillating Surface Pressure Distributions, Journal of Fluid Mechanics, 114, p. 467-485, 1982.

7. Evans D. V. and Porter R., Hydrodynamic Characteristics of an Oscillating Water Column Wave Energy Device, Applied Ocean Research 17, p. 155-164, 1995.

8. Evans D. V. and Porter R., Efficient Calculation of , Hydrodynamic Properties of OWC-type devices, J. Offshore Mech. Arctic Eng. 119, p. 210-218, 1997.

9. Kim T., Takao M., Setoguchi T., Kaneko K. and Inoue M., Performance comparison of turbines for wave power conversion, International Journal of Thermal Sciences 40, $\mathrm{p}$. 681-689, 2001.

10. Setoguchi T., Santhakumar S., Takao M., Kim T. H. and Kaneko K., A modified Wells turbine for wave energy conversion, Renewable Energy 28, p. 79-91, 2003.

11. De O. Falcão A. F., Control of an oscillating-water-column wave power plant for maximum energy production, Applied Ocean Research 24, p. 73-82, 2002.

12. El Marjani A., Castro Ruiz F., Rodriguez M. A., Para Santos M. T., Numerical modelling in wave energy conversion systems, Energy 33, p. 1246-1253, 2008.

13. Paixãdo Conde J. M., Gato L. M. C., Numerical study of the air-flow in an oscillating water column wave energy converter, Renewable Energy 33, p. 2637-2644, 2008.

14. White F. M., Fluid Mechanics, New York, McGraw-Hill, 2001.

15. McCormick M. E., Ocean Wave Energy Conversion, New York, John Wiley\&Sons, 1983.

16. Fluent Version 6.3 Tutorial 10, Simulation of wave generation in a tank, Fluent Inc., 2005, p. 10.1-10.23.

17. Fluent Version 6.3 User's Guide, Fluent Inc., 2006, p.25.33-25.36. 